ARTICLE

DOI: $10.1038 /$ ncomms10659

OPEN

\title{
Hexadecapolar colloids
}

Bohdan Senyuk ${ }^{1}$, Owen Puls', Oleh M. Tovkach ${ }^{2,3}$, Stanislav B. Chernyshuk ${ }^{4}$ \& Ivan I. Smalyukh $1,5,6$

Outermost occupied electron shells of chemical elements can have symmetries resembling that of monopoles, dipoles, quadrupoles and octupoles corresponding to filled $s-, p-, d-$ and $f$ orbitals. Theoretically, elements with hexadecapolar outer shells could also exist, but none of the known elements have filled $g$-orbitals. On the other hand, the research paradigm of 'colloidal atoms' displays complexity of particle behaviour exceeding that of atomic counterparts, which is driven by DNA functionalization, geometric shape and topology and weak external stimuli. Here we describe elastic hexadecapoles formed by polymer microspheres dispersed in a liquid crystal, a nematic fluid of orientationally ordered molecular rods. Because of conically degenerate boundary conditions, the solid microspheres locally perturb the alignment of the nematic host, inducing hexadecapolar distortions that drive anisotropic colloidal interactions. We uncover physical underpinnings of formation of colloidal elastic hexadecapoles and describe the ensuing bonding inaccessible to elastic dipoles, quadrupoles and other nematic colloids studied previously.

\footnotetext{
${ }^{1}$ Department of Physics and Soft Materials Research Center, University of Colorado, Boulder, Colorado 80309, USA. ${ }^{2}$ Bogolyubov Institute for Theoretical Physics, NAS of Ukraine, Kyiv 03680, Ukraine. ${ }^{3}$ Department of Mathematics, University of Akron, Akron, Ohio 44325, USA. ${ }^{4}$ Institute of Physics, NAS of Ukraine, Kyiv 03650, Ukraine. ${ }^{5}$ Department of Electrical, Computer, and Energy Engineering, Materials Science and Engineering Program, University of Colorado, Boulder, Colorado 80309, USA. ${ }^{6}$ Renewable and Sustainable Energy Institute, National Renewable Energy Laboratory and University of Colorado, Boulder, Colorado 80309, USA. Correspondence and requests for materials should be addressed to I.I.S. (email: ivan.smalyukh@colorado.edu).
} 
C olloids form a platform for scalable fabrication of mesostructured composite materials and provide a framework for testing theoretical descriptions of crystals and glasses, albeit they are also commonly encountered in daily life in forms of milk, paints, coffee, fog and so on ${ }^{1-5}$. Nematic liquid crystal (NLC) colloids ${ }^{6-17}$ attract particularly strong interest because they can be tuned by weak external stimuli, such as lowvoltage fields and light, similar to the NLC host fluids themselves ${ }^{12,17}$. Colloidal particles locally perturb the uniform ground-state alignment of the anisotropic NLC molecules described by the so-called 'director' $\mathbf{n} \equiv-\mathbf{n}$ with non-polar symmetry, producing spatial elastic distortions of the director field $\mathbf{n}(\mathbf{r})$ that resemble electric field configurations around dipolar and quadrupolar charge distributions ${ }^{7-12}$. The type of distortions depends on surface boundary conditions for $\mathbf{n}(\mathbf{r})$, which can be tangential or perpendicular to the particle surfaces and can induce both bulk and surface line and point defects $^{7-12}$, topological singularities along which $\mathbf{n}(\mathbf{r})$ and NLC order cannot be defined ${ }^{1}$. Such NLC colloidal particles tend to arrange themselves so that they can share energetically costly $\mathbf{n}(\mathbf{r})$ distortions to minimize free energy, exhibiting highly anisotropic elasticity-mediated interactions that resemble interactions of electrostatic dipoles and quadrupoles ${ }^{6-12,15,16}$. This electrostatics analogy provides a framework for understanding, predicting and engineering the ensuing colloidal self-assembly. However, for many years of very active research efforts ${ }^{6-12,15,16}$, only colloidal elastic dipoles and quadrupoles have been found in experiments and theories alike, which limits diversity of the accessible colloidal bonds and self-assembled structures ${ }^{6-17}$.

In this work, we describe colloidal elastic hexadecapoles that spontaneously form around solid microspheres immersed in a uniformly aligned NLC. The unusual symmetry of elastic distortions arises from conically degenerate ${ }^{18}$ boundary conditions for $\mathbf{n}(\mathbf{r})$ at the colloidal surfaces, which induce surface point and line defects at the same time. Using a combination of holographic optical tweezers (HOT) ${ }^{19}$, nonlinear optical imaging ${ }^{20}$ and polarizing optical microscopy $(\mathrm{POM})^{1,6}$, we probe the $\mathbf{n}(\mathbf{r})$-distortions and quantify colloidal pair interactions by measuring distance and angular dependencies of elastic potentials, demonstrating relations between the director structure and medium-mediated inter-particle forces. Finally, we explain our findings using a model based on elastic multipole expansion and discuss how the experimental framework we have developed may enable colloidal self-assembly into novel forms of tunable pre-engineered matter without known atomic analogues.

\section{Results}

Hexadecapolar elastic multipole. When dispersed in a uniformly aligned NLC fluid host, polystyrene microspheres (PSMs) of a radius $r_{0}$ locally distort $\mathbf{n}(\mathbf{r})$, which is manifested by eight bright lobes around the particle perimeter seen in POM between the crossed polarizer and analyser (Fig. 1a). These bright lobes are separated by eight dark regions within which $\mathbf{n}(\mathbf{r})$ at the particle's perimeter is parallel to polarizer or analyser. Using a phase retardation plate and interference of polarized light propagating through the particle-distorted structure, we reveal that $\mathbf{n}(\mathbf{r})$ tilting away from the far-field director $\mathbf{n}_{0}$ switches between clockwise and counterclockwise directions (corresponding to the blue and yellow colours in the micrograph) eight times as one circumnavigates the sphere (Fig. 1b). Bright-field micrographs obtained at different depth of focus reveal presence of weakly scattering surface point defects (boojums) at the particle poles along $\mathbf{n}_{0}$ as well as a circular loop of a defect line (often called 'Saturn ring' $)^{9,10}$ at the particle's equator (Fig. 1c,e). Based on POM and three-dimensional nonlinear optical imaging (Fig. 1a-c,e and
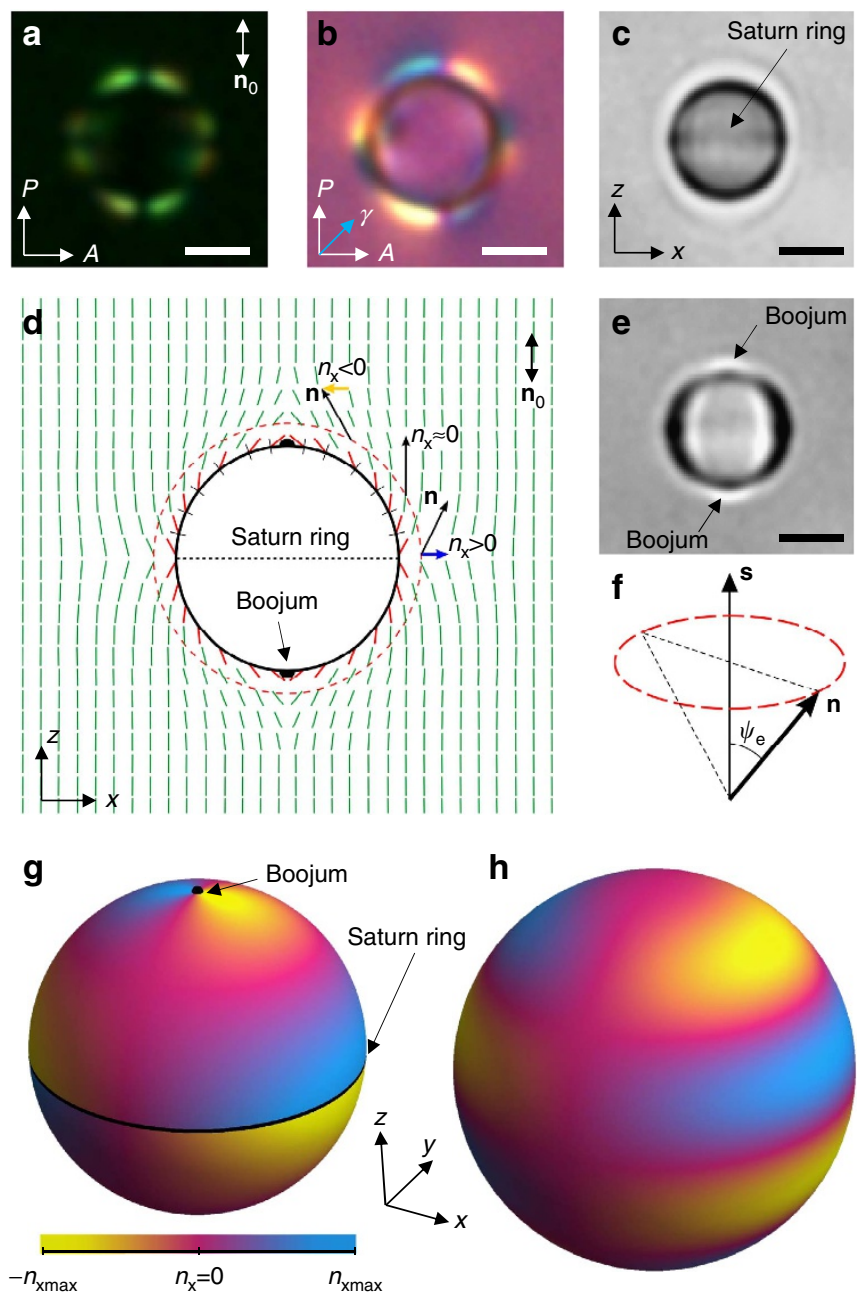

Figure 1 | Elastic hexadecapole induced by a colloidal PSM. (a-d) Optical micrographs obtained with $(\mathbf{a}, \mathbf{b})$ POM and (c,e) bright field microscopy. $P, A$ and $\gamma$ mark the crossed polarizer, analyser and a slow axis of a $530 \mathrm{~nm}$ retardation plate (aligned at $45^{\circ}$ to $P$ and $A$ ), respectively. (d) Schematic diagram of induced $\mathbf{n}(\mathbf{r})$ (green rods) satisfying the tilted boundary conditions at the PSM surface (red rods), with the 'easy axis' at a constant angle $\psi_{\mathrm{e}}$ to a local normal $\mathbf{s}$ to the surface (black rods). (f) Schematic of conic degenerate surface boundary conditions. (g,h) Three-dimensional visualization of the $x$-component $n_{x}$ of $\mathbf{n}(\mathbf{r})(\mathbf{g})$ at the surface of PSM for $\psi_{\mathrm{e}}=45^{\circ}$ and $(\mathbf{h})$ at a spherical surface of radius $1.2 r_{0}$ shown using a dashed red circle in $\mathbf{d}$. Blue, yellow and magenta colours correspond, respectively, to a positive, near-zero and negative $n_{x}$ according to the colour scheme shown in $\mathbf{g}$. Dashed equatorial line in $\mathbf{d}$ and a black solid line in $\mathbf{g}$ depict the 'Saturn ring' surface defect loop at the particle's equator visible in c. Black hemispheres in $\mathbf{d}$ and $\mathbf{g}$ show surface point defects boojums at the poles of the particle visible in e. Scale bars, $2 \mu \mathrm{m}$.

Supplementary Figs 1-3), we uncover the structure of $\mathbf{n}(\mathbf{r})$ distortions schematically shown in Fig. 1d. This structure is consistent with conically degenerate surface boundary conditions for $\mathbf{n}(\mathbf{r})$ with respect to PSM's local surface normals $\mathbf{s}$, which were previously demonstrated for NLCs at flat polystyrene-coated surfaces ${ }^{18}$. The director's easy axis orientation lies on a cone of equilibrium polar angle $\psi_{\mathrm{e}}$ (Fig. 1f). To minimize the free-energy cost of bulk elastic distortions, interaction of conically degenerate surface boundary conditions on the microsphere with the uniform $\mathbf{n}_{0}$ lifts this conical degeneracy and yields an axially symmetric $\mathbf{n}(\mathbf{r})$ depicted in Fig. 1d. Extending this analysis to three dimensions, the projection $n_{x}$ of $\mathbf{n}(\mathbf{r})$ onto the $x$-axis 
orthogonal to $\mathbf{n}_{0}$ can be visualized around PSM using colours that highlight positive, near zero and negative $n_{x}$ (Fig. 1g). The black points at the poles and ring at the equator of the sphere are regions of discontinuity of $\mathbf{n}(\mathbf{r})$ at the NLC-PSM interface and correspond to the boojum and Saturn ring topological defects, respectively. Away from the particle surface and these topological singularities, the experimentally reconstructed $\mathbf{n}(\mathbf{r})$ is continuous (Fig. 1d) and consistent with the theoretical model for a hexadecapolar director distortions presented in a similar way in Fig. $1 \mathrm{~h}$.

Elastic interactions between hexadecapolar colloids. Elasticitymediated interactions between PSMs (Fig. 2) differ from all NLC
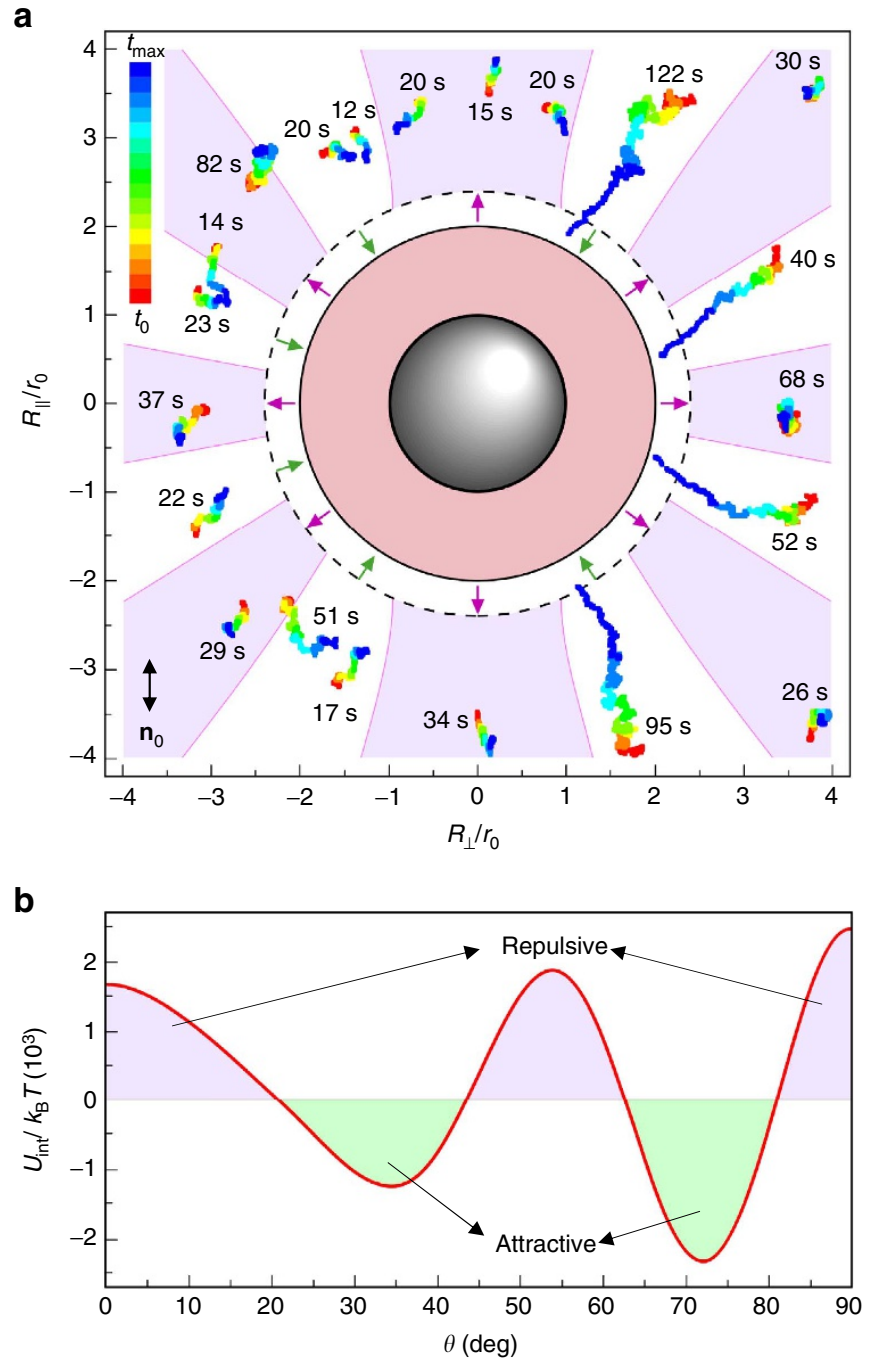

Figure 2 | Elastic interactions of colloidal hexadecapoles. (a) Angular dependence of interactions is probed by tracking motion of a particle released from the optical trap and moving with respect to the 'stationary' trapped particle in the centre depending on the orientation of $\mathbf{R}$ with respect to $\mathbf{n}_{0}$. The elapsed time is coded according to the colour scale (inset) and the maximum elapsed times $t_{\max }-t_{0}$ are marked next to the corresponding trajectories. $R_{\|}$and $R_{\perp}$ denote the centre-to-centre distance $\mathbf{R}$ components along and perpendicular to $\mathbf{n}_{0}$, respectively. The PSM of radius $r_{0}$ is surrounded by the spherical volume of radius $2 r_{0}$ that is excluded for centres of other PSMs. The nonlinear zone is shown by a dashed circle at $R=2.4 r_{0}$. (b) Pair-potential $U_{\text {int }}$ versus $\theta$ for two particles at $R=2.4 r_{0}$. Angular zones of repulsion and attraction are highlighted using magenta and green arrows and colouring, respectively. colloids studied so far. To get insights into the strength and direction-dependence of these interactions, we utilize HOT to optically trap one 'stationary' PSM and then release another particle at different centre-to-centre vector $\mathbf{R}$ orientations with respect to $\mathbf{n}_{0}$ as well as at different separation distances. Using video-microscopy, we track the ensuing particle motions, which result from a combination of Brownian jiggling and elasticitymediated interactions. The submicron waist and relatively low power $(\sim 10 \mathrm{~mW})$ of a focused trapping beam allow us to avoid the influence of the trapping on the measurement ${ }^{21}$. Furthermore, the laser tweezers are used only to bring the particles to the desired initial conditions and are turned off within the time when the pair-interaction is probed with video microscopy, allowing us to avoid possible artefacts associated with the complex effects of the laser trapping light at small interparticle distances. The colour-coded time-coordinate trajectories of particles released from optical traps at different $\mathbf{R}$ are shown in Fig. 2a. Unlike in the case of dipolar and quadrupolar NLC colloids $7,8,15,16$, elastic forces are relatively short-ranged and exceed the strength of thermal fluctuations only at distances of four-to-five particle radii $r_{0}$. However, the angular dependence of these forces is very rich, with eight angular segments of inter-particle attractions separated by eight angular segments of repulsions, with the intermediate angular sectors within which particles move sideways as the inter-particle elastic forces are orthogonal or at large angles to $\mathbf{R}$ (Fig. 2a). These angular sectors of attraction and repulsion correlate with the bright and dark regions of POM micrographs (Fig. 1a) as well as with the predictions of our model based on elastic multipole expansion (Fig. 2).

To quantify elastic interactions, we first probe the centre-tocentre separation $R=|\mathbf{R}|$ versus time $t$ for particles released at different angles between $\mathbf{R}$ and $\mathbf{n}_{0}$ within the angular sectors of attraction (Fig. 3a) and then calculate particle velocities $\dot{\mathbf{R}}=d \mathbf{R} / d t$. Because the system is highly over-damped and the inertia effects can be neglected ${ }^{22}$, the experimentally measured $\mathbf{R}(t)$ and the simplified equation of motion $0 \approx-\xi \dot{\mathbf{R}}+\mathbf{F}_{\text {int }}$ yield the pair interaction potential $U_{\text {int }}$ (inset of Fig. 3a), where $\xi$ is a drag coefficient measured separately by characterizing Brownian motion of PSMs (Methods and Supplementary Fig. 4) and $\mathbf{F}_{\text {int }}=-\nabla U_{\text {int }}$ is the elastic interaction force. The attractively interacting particles stop short of physically touching each other, instead forming stable dimer assemblies with typical $R \approx(2.05-2.2) r_{0}$ and stable $\mathbf{R}$ orientations with respect to $\mathbf{n}_{0}$ within one of the two angular sectors of assembly in each quadrant dependent on $\psi_{\mathrm{e}}: \theta_{1} \approx 22^{\circ}-26^{\circ}$ or $\theta_{2} \approx 64^{\circ}-75^{\circ}$ (Fig. 3b-f). Multi-particle assemblies with different combinations of angles $\theta_{1}$ and $\theta_{2}$ are also observed (Fig. 3d). The inter-particle binding energies are measured to be in the range of hundreds of $k_{\mathrm{B}} T$ (inset of Fig. 3a), making them robust with respect to thermal fluctuations. Although $U_{\text {int }}$ versus $\theta$ has eight minima, only four of them can be occupied simultaneously in one plane by particles of the same size because of the 'excluded volume' effects, yielding two-dimensional colloidal crystals with rhombic elementary cells (Supplementary Fig. 5 and Supplementary Note 1). Following similar considerations, a large number of low-symmetry threedimensional colloidal structures can be envisaged too. Since the elastic interactions potential is hundreds of $k_{\mathrm{B}} T$ and the particle assemblies can be entrapped in metastable states, the assembly of two- and three-dimensional colloidal lattices from micrometersized particles requires the use of optical tweezers for guiding colloidal particles. Alternatively, the elastic interaction potentials between colloidal particles of smaller size or with weaker surface anchoring can be brought to the order of $10 k_{\mathrm{B}} T$ and lower, so that the crystal self-assembly can occur without the assistance of optical tweezers, which will be explored elsewhere. 
a
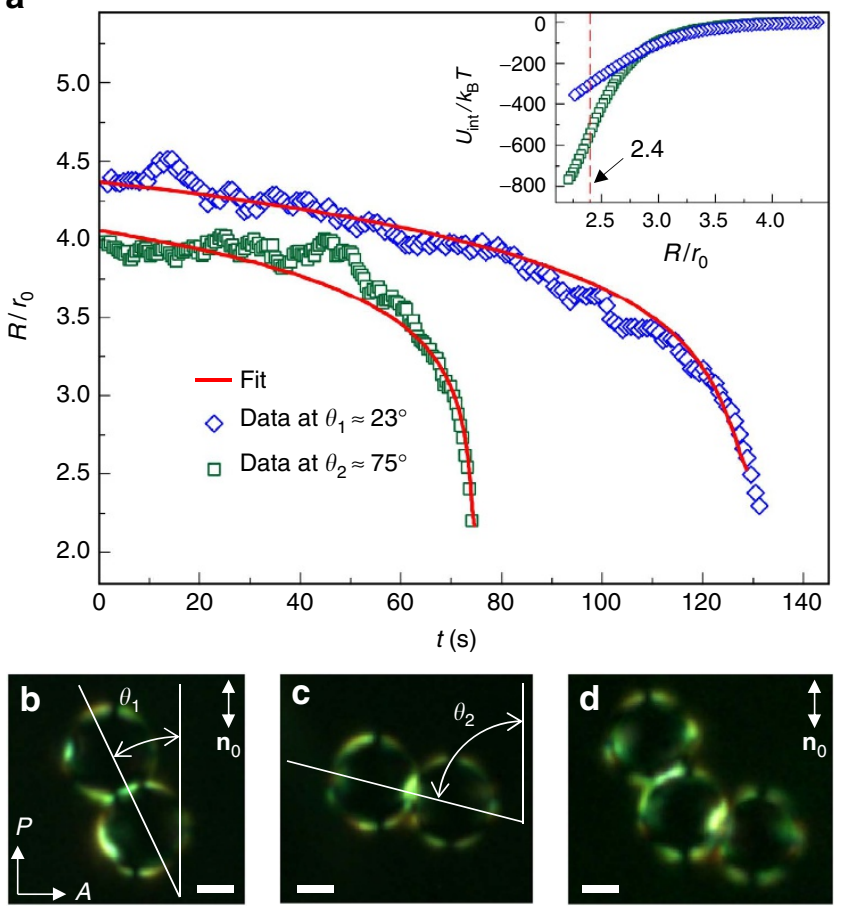

e

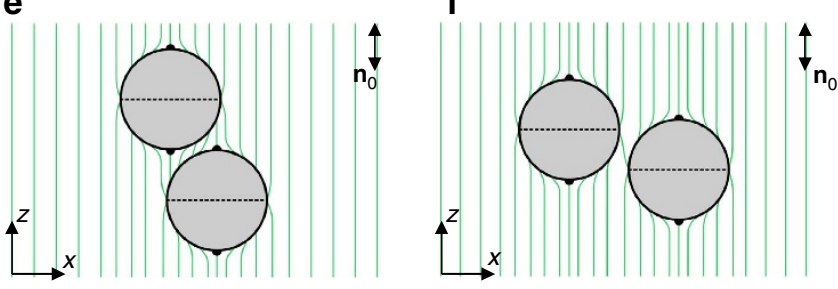

Figure 3 | Self-assembly of hexadecapolar colloids. (a) Separation $R$ versus time $t$ for attractive interactions at different $\theta$ (measured upon the dimer formation). The solid red line shows a least-squares fit with $R(t)$ obtained from the simplified equation of motion with $\left(b_{2}, b_{4}, b_{6}\right)=$ $(-0.017,-0.092,0.003)$; it is impossible to reproduce such a dependence $R(t)$ with the parameter $b_{2}$ only since the quadrupolequadrupole interaction is repulsive along $\theta \approx 23^{\circ}$. Inset shows the corresponding $U_{\text {int }}$ versus $R$. (b) and (c) POM micrographs of selfassembled colloidal dimers of hexadecapoles with $\mathbf{R}$ aligned at $\theta_{1}$ and $\theta_{2}$ to $\mathbf{n}_{0}$, respectively. (d) POM micrograph of a kinked colloidal chain of hexadecapoles. (e,f) Schematic diagrams of $\mathbf{n}(\mathbf{r})$ (green lines) for colloidal dimers shown in $\mathbf{c}, \mathbf{d}$, respectively. Dashed lines and black hemispheres in c and $\mathbf{d}$ depict surface defect loops and surface point defects at the poles of PSMs, respectively. Scale bars, $2 \mu \mathrm{m}$.

To model experiments, we exploit the electrostatic analogy of the far-field director distortions because of a colloidal sphere that can be represented as elastic multipoles ${ }^{23}$, albeit our colloids dramatically differ from elastic dipoles and quadrupoles studied so $\mathrm{far}^{7-12,23-26}$. Far from the colloidal sphere, the director deviations $n_{\mu}(\mu=x ; y)$ from $\mathbf{n}_{0}=(0,0,1)$ are small. Assuming $\mathbf{n}(\mathbf{r}) \approx\left(n_{x}, n_{y}, 1\right)$, the NLC elastic free-energy reads $s^{7,8,23}$

$$
F_{\text {har }}=\frac{K}{2} \sum_{\mu=x, y} \int d \mathbf{r} \nabla n_{\mu} \cdot \nabla n_{\mu}
$$

where $K$ is an average Frank elastic constant ${ }^{1}$. Euler-Lagrange equations arising from the functional (1) are of Laplace type,
$\Delta n_{\mu}=0$, with solutions expanded into multipoles

$$
n_{\mu}(\mathbf{r})=\sum_{l=1}^{N} a_{l}(-1)^{l} \partial_{\mu} \partial_{z}^{l-1} \frac{1}{r}
$$

where $a_{l}=b_{l} r_{0}{ }^{l+1}$ is the elastic multipole moment of the $l$ th order $\left(2^{l} \text {-pole }\right)^{22-26}$ and one can find coefficients $b_{l}$ from exact solutions for $\mathbf{n}(\mathbf{r})$ or from relevant experiments (Figs $2 \mathrm{a}$ and $3 \mathrm{a}$ ). Odd moments vanish because $\mathbf{n}(\mathbf{r})$ is symmetric about the particle centre, similar to analogous electrostatic charge distributions that have no dipole or octupole electric moments ${ }^{22-26}$. The multipole expansion of the induced $\mathbf{n}(\mathbf{r})$ shown in Fig. 1d is characterized by coefficients $b_{2}, b_{4}$ and $b_{6}$, which also determine the colloidal pair-interaction energy ${ }^{24}$

$$
U_{\text {int }}=4 \pi K \sum_{l, l^{\prime}=2,4,6} a_{l} a_{l^{\prime}}^{\prime}(-1)^{l^{\prime}} \frac{\left(l+l^{\prime}\right) !}{R^{l+l^{\prime}+1}} P_{l+l^{\prime}}(\cos \theta),
$$

where $P_{l+l^{\prime}}(\cos \theta)$ are the Legendre polynomials. For colloidal quadrupoles, $b_{2}$ dominates and $b_{4}$ and $b_{6}$ can play a role only at small $^{25} R$. For our particles (Fig. 1), the induced $\mathbf{n}(\mathbf{r})$ can be qualitatively understood as a superposition of configurations of two separate quadrupoles, one with the Saturn-ring and one with boojums ${ }^{9-11,16,22-26}$, having opposite signs of quadrupole moments (compare Fig. $4 \mathrm{a}, \mathrm{d}, \mathrm{g}, \mathrm{j}$ and $4 \mathrm{~b}, \mathrm{e}, \mathrm{h}, \mathrm{k}$ ). Therefore, the net quadrupole moment is small and the high-order multipoles manifest themselves in a wide range of $R$. Fitting experimental $R(t)$ within different angular sectors of pair interaction with the corresponding theoretical predictions yields a unique set of parameters $b_{2}, b_{4}$ and $b_{6}$ (Fig. 3a). The quadrupole moment $a_{2}=-0.017 r_{0}{ }^{3}$ is about two orders of magnitude smaller than that of elastic quadrupoles $^{16,24}$, consistent with the hexadecapolar symmetry of $\mathbf{n}(\mathbf{r})$ that is found playing a dominant role, and even the higherorder term (64-pole) plays a detectable role at relatively small $R$ (Supplementary Fig. 6 and Supplementary Note 2). The rich angular dependence of elastic pair-interactions predicted by equation 3 for coefficients $b_{2}, b_{4}$ and $b_{6}$ obtained from fitting is consistent with our experimental characterization of the hexadecapolar nature of PSM colloids in the NLC host (Fig. 2).

\section{Discussion}

It is instructive to compare hexadecapolar NLC colloids to other known elastic multipoles and their electrostatic analogues. Colloidal spheres with strong tangential anchoring at their surface form $\mathbf{n}(\mathbf{r})$-distortions of quadrupolar configuration $^{16,24,27,28}$ and are commonly called elastic quadrupoles (Fig. 4a,d,g,j). They have two surface point defects at the two poles of the spherical particle, which are called 'boojums'. Spherical particles with homeotropic surface anchoring can induce two different elastic multipoles, a dipole $\mathrm{e}^{23,28}$ (Fig. 4c,f,l) and a quadrupole $\mathrm{e}^{16,23,27-32}$ (Fig. 4b,e,h,k). The elastic colloidal dipole has a bulk point defect called 'hedgehog' near one of the poles and lacks mirror symmetry with respect to the plane going through its equator orthogonally to $\mathbf{n}_{0}$ (Fig. 4 f,l). Such a colloidal dipole has a small octupole moment as well ${ }^{33}$. The elastic quadrupole around the particle with a strong homeotropic anchoring has a closed disclination loop around the equator called 'Saturn ring' and, in all four quadrants, has an opposite tilt of the deformed director with respect to $\mathbf{n}_{0}$ as compared with the tangential elastic quadrupole ${ }^{16}$ (compare Fig. $4 \mathrm{~g}, \mathrm{j}, \mathrm{h}, \mathrm{k}$ ). In the case when anchoring is weak, an elastic multipole with weak distortions of quadrupolar configuration can be formed somewhat similar to the elastic quadrupole with 'Saturn ring', but its disclination loop is 'virtual' (within particle's volume) as the director is allowed to deviate away from the easy axis orientation $^{31,32}$. The maps of $x$-component of the director, $n_{x}$, calculated using methods described in refs 23,24 and plotted on 


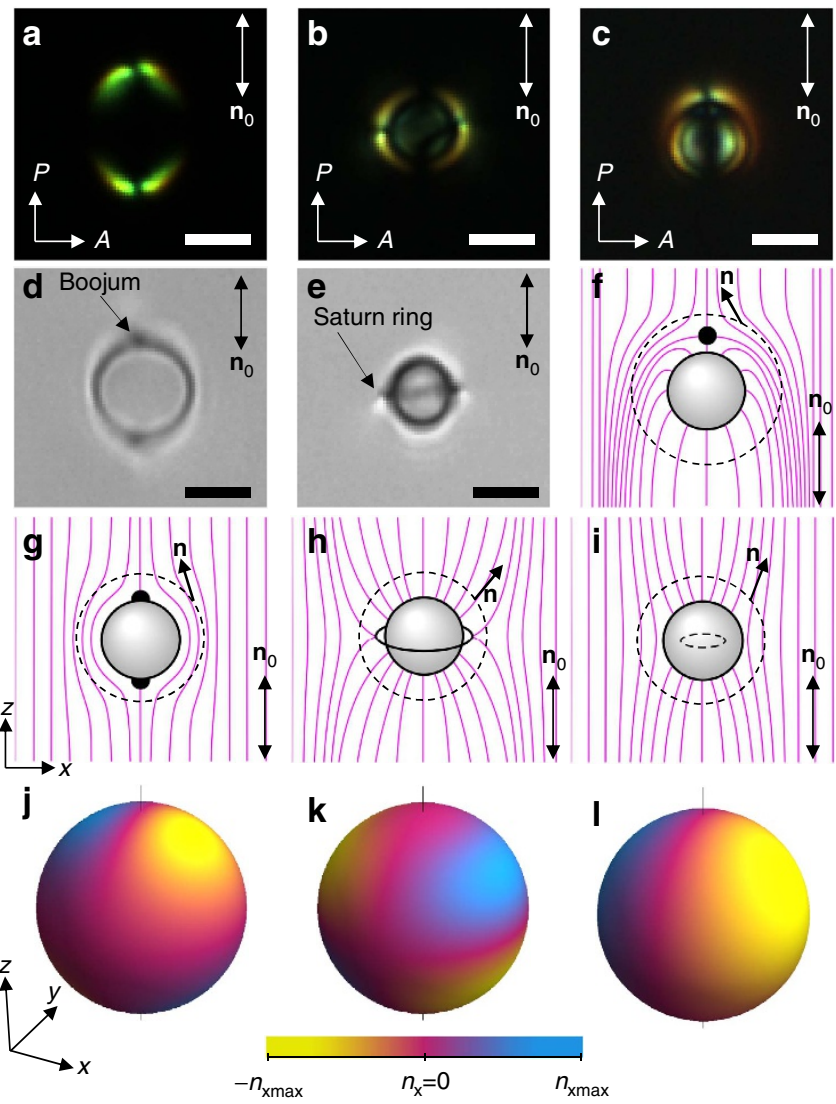

Figure 4 | Elastic multipoles induced by spherical particles with different anchoring. $(\mathbf{a}, \mathbf{b})$ Polarizing and $(\mathbf{d}, \mathbf{e})$ bright field micrographs and $(\mathbf{g}, \mathbf{h})$ schematic diagrams of a director field $\mathbf{n}(\mathbf{r}$ ) (magenta lines) around elastic quadrupoles, respectively, formed by spheres with a strong $(\mathbf{a}, \mathbf{d}, \mathbf{g})$ tangential and $(\mathbf{b}, \mathbf{e}, \mathbf{h})$ homeotropic anchoring. Black filled semicircles at the poles of the particle in $\mathbf{g}$ show surface point defects dubbed 'boojums.' A line at the particle equator in $\mathbf{h}$ shows a closed surface disclination loop dubbed 'Saturn ring.' (c) Polarizing micrograph and (f) a schematic diagram of $\mathbf{n}(\mathbf{r})$ around an elastic dipole formed by a sphere with a strong homeotropic surface anchoring. A black point in the front of the particle shown schematically in $\mathbf{f}$ depicts a bulk point defect 'hedgehog.'

(i) Schematic diagram of a quadrupolar $\mathbf{n}(\mathbf{r})$ around a sphere with weak surface anchoring. A dashed loop inside the particle shows a virtual disclination ring. (j-I) Colour-coded visualizations of the $n_{x}$ component of $\mathbf{n}(\mathbf{r})$, which is caused by the tilt of $\mathbf{n}(\mathbf{r})$ away from $\mathbf{n}_{0}$, at the spherical surface enveloping the particles shown, respectively, in $\mathbf{f , g}, \mathbf{h}$. The spherical surfaces of colour-coded visualization of director distortions have radius of $(1.2-1.4) r_{0}$, as shown by dashed circles in $\mathbf{f}-\mathbf{h}$. Blue, yellow and magenta colours correspond, respectively, to a positive, zero and negative $n_{x}$ according to a colour scale bar provided in $\mathbf{k}$. Scale bars, $4 \mu \mathrm{m}$.

spherical surfaces encompassing the particles and the defects, clearly illustrate the multipolar nature of elastic distortions similar to that in the electrostatics analogues ${ }^{34,35}$. A direct comparison of the symmetry of the director distortions around elastic dipoles and quadrupoles with that of hexadecapolar PSMs in NLCs helps to highlight the very different nature of these highorder NLC colloidal multipoles studied in our work (compare Figs $4 \mathrm{j}-\mathrm{l}$ and $1 \mathrm{~h}$ ). On the other hand, the hexadecapolar NLC colloids further expand the analogy between electrostatic and elastic colloidal multipoles. In fact, the colour presentations of elastic distortions induced by colloidal dipoles (Fig. 4l), two different quadrupoles (Fig. 4j,k) and our hexadecapoles (Fig. 1g,h) resemble very closely the corresponding dipolar, quadrupolar and hexadecapolar electrostatic charge distributions on a sphere described by a spherical harmonic function ${ }^{35}$ $\sigma_{l}^{m}(\Theta, \phi)=N \cos (m \phi) P_{l}^{m}(\cos \Theta)$ with $(l, m)=(1,1),(2, \pm 1)$ and $(4,1)$, respectively, where $N$ is a normalization constant, $\Theta$ is a polar and $\phi$ is an azimuthal angles, $P_{l}^{m}(\cos \Theta)$ is the associated Legendre polynomial; the constant $l$ determines the order of a multipole (that is, $2^{l}$-th pole) and $-l \leq m \leq l$. This close analogy between electrostatic and elastic colloidal multipoles may help devising approaches for self-assembly of colloidal mesostructured composite materials.

To conclude, we describe hexadecapolar NLC colloids with unusual field configurations, highly anisotropic elastic interactions, and versatile forms of self-assembly. Our findings pose a challenge of realizing pure NLC colloidal octupoles, lower order multipoles that have not been observed so far, and provide the means of probing the role of hexadecapolar moments in inter-molecular interactions using colloidal model systems. Self-assembly of hexadecapolar NLC colloids is expected to yield a diverse family of two- and three-dimensional low-symmetry crystal lattices. Beyond the rich experimental platform for fundamental studies, our colloids have potential uses in designing and realizing reconfigurable 'soft' photonic crystals ${ }^{36}$ and other NLC-based mesostructured composites ${ }^{37}$ with properties that can be pre-engineered through controlling tilted boundary conditions on nanoparticle surfaces ${ }^{38-40}$, particle shape, composition and topology, $2,6,8,41$ as well as the NLC host properties, particle active behaviour $^{42}$ and the use of external stimuli ${ }^{12,17}$

\section{Methods}

Preparation of nematic colloidal dispersions. Colloidal PSM particles of radii $r_{0} \approx 2.05,2.65$ and $4 \mu \mathrm{m}$ and with conically degenerate surface boundary conditions were prepared using one of two different methods as follows ${ }^{43,44}$. In the first method, asymmetric colloidal polystyrene dimers ${ }^{43,45}$ were synthesized using a modified seeded polymerization technique ${ }^{44-46}$ (Supplementary Fig. 1a). Resulting dimers in ethanol were sonicated for tens of minutes using an ultrasonic bath 8891 (Cole-Parmer) to break apart large and small spherical lobes forming the asymmetric colloidal dimer (Supplementary Fig. 1b,c). Colloidal spheres originating from these dimers were then re-dispersed in pentyl-cyano biphenyl (5CB) obtained from Frinton Laboratories, Inc. The larger (diameter $D=2 r_{0} \approx 4.1 \mu \mathrm{m}$ ) colloidal spheres, which were used as PSMs in our experiments intrinsically provide conic degenerate surface boundary conditions for the NLC director. In the second preparation method, we used polystyrene divinylbenzene spherical particles PC06N (Bang Laboratories, Inc.) of $D \approx 8 \mu \mathrm{m}$ and DC-05 (Thermo Scientific) of $D \approx 5.3 \mu \mathrm{m}$ dispersed in $5 \mathrm{CB}$. As dispersed, these particles provide tangential alignment for $5 \mathrm{CB}$ molecules and the director field $\mathbf{n}(\mathbf{r})$. However, after keeping these colloidal dispersions in $5 \mathrm{CB}$ at the elevated temperature of $\approx 100^{\circ} \mathrm{C}$ for about $12 \mathrm{~h}$, the alignment at the surface of these polymer particles changed to a conical degenerate (Supplementary Fig. 1d-f), similar to that reported in ref. 18 for flat surfaces coated by polystyrene. The tilt angle of the easy axis $\psi_{\mathrm{e}}$ was varying approximately within $20^{\circ}-75^{\circ}$, depending on details of preparation. The comparison of the surface free-energy density $F_{s}=W_{c}\left(\cos ^{2} \psi-\cos ^{2} \psi_{\mathrm{e}}\right)^{2} / 2$ costs (ref. 18) for deviating $\mathbf{n}(\mathbf{r})$ to a polar angle $\psi$ away from the easy axis at $\psi_{\mathrm{e}}$ relative to the bulk elastic energy density is characterized by the so-called conical anchoring extrapolation length $K / W_{c} \sim(50-500) \mathrm{nm} \ll r_{0}$, where ${ }^{18} W_{c} \sim 10^{-5} \mathrm{Jm}^{-2}$. Since $K / W_{c} \ll r_{0}$, one can assume that the tilted conically degenerate boundary conditions are rigid with respect to changing $\psi$. To prepare samples with NLC dispersions in a uniformly aligned host, dry PSMs were added to $5 \mathrm{CB}$. The dilute NLC colloidal dispersions were then filled into the cells comprised of two glass plates separated by glass spacers defining the cell gap in the range $15-30 \mu \mathrm{m}$, which were sealed at all edges using fast-setting epoxy glue after the filling process. The inner surfaces of cell substrates were treated with polyimide PI2555 (obtained from HD Microsystems) for in-plane homogeneous alignment of the far-field director $\mathbf{n}_{0}$ defined by unidirectional rubbing.

Imaging techniques and data acquisition. POM (Fig. 1 and Supplementary Figs 1,2), nonlinear optical imaging (Supplementary Fig. 3) and laser manipulations of PSM particles were performed using a single integrated setup capable of simultaneous conventional optical and nonlinear three-photon excitation fluorescence polarizing microscopy (3PEF-PM) ${ }^{20}$ and HOT optical control operating at $1,064 \mathrm{~nm}$ (ref. 19). The setup was built around an inverted microscope IX81 (Olympus). For 3PEF-PM imaging ${ }^{20}$, we have employed a tunable $(680-1,080 \mathrm{~nm})$ Ti-Sapphire oscillator (Chameleon Ultra II, Coherent) emitting $140 \mathrm{fs}$ pulses at a repetition rate of $80 \mathrm{MHz}$. The laser wavelength was tuned to $870 \mathrm{~nm}$ for the 
three-photon excitation of 5CB molecules and the 3PEF-PM signals were collected in epi-detection mode with a photomultiplier tube (H5784-20, Hamamatsu). An Olympus $\times 100$ oil-immersion objective with a high numerical aperture of 1.4 was used for both optical imaging and laser trapping. This experimental setup is described in details elsewhere ${ }^{19,20}$. Motion of colloidal particles was recorded with a charge-coupled camera (Flea, PointGrey) at a rate of $15 \mathrm{fps}$ and their lateral positions versus time were determined from captured sequences of images using motion tracking plugins of ImageJ software (obtained from National Institutes of Health) with the accuracy of 7-10 nm.

Measuring anisotropic diffusion of elastic NLC hexadecapoles. PSMs with conic anchoring distort the homogeneous $\mathbf{n}(\mathbf{r})$ and form elastic hexadecapoles in the aligned NLC (Fig. 1a-e and Supplementary Figs 1b,d-f, 2,3b). These hexadecapoles stay suspended in the bulk of NLC (Supplementary Fig. 3b), which is facilitated by elastic repulsion of PSMs from the confining substrates and their Brownian motion because of thermal fluctuations. Using bright field microscopy and video tracking, one can determine a position of the particle within each frame and then analyse its translational displacements at regular time steps corresponding to the video frame rates. Following the well-established methodology ${ }^{29}$ and using the histograms of displacements shown in Supplementary Fig. 4, it is possible to experimentally determine two independent diffusion coefficients $D_{\|}=1.63 \times 10^{-3} \mu \mathrm{m}^{2} \mathrm{~s}^{-1}$ and $D_{\perp}=1.05 \times 10^{-3} \mu \mathrm{m}^{2} \mathrm{~s}^{-1}$, which characterize, respectively, the diffusion of elastic hexadecapoles along and perpendicular to the far-field director $\mathbf{n}_{0}$. As $D_{\|}>D_{\perp}$, it is easier for the NLC hexadecapoles to move along $\mathbf{n}_{0}$ than in other directions. The ratio $D_{\|} / D_{\perp}=1.55$ is not much different from values determined for other elastic multipoles studied previously ${ }^{29,30}$ (Fig. 4). These coefficients are also used to determine friction coefficients from the StokesEinstein relation, which are then subsequently used in the calculation of anisotropic interaction forces ${ }^{27}$ between hexadecapoles.

\section{References}

1. Chaikin, P. M. \& Lubensky, T. C. Principles of Condensed Matter Physics (Cambridge University Press, 2000).

2. Manoharan, V. N. Colloidal matter: packing, geometry, and entropy. Science 349, 1253751 (2015).

3. Anderson, V. J. \& Lekkerkerker, H. N. W. Insights into phase transition kinetics from colloid science. Nature 416, 811-815 (2002).

4. Sacanna, S., Irvine, W. T. M., Chaikin, P. M. \& Pine, D. J. Lock and key colloids. Nature 464, 575-578 (2010).

5. Jones, M. R. et al. DNA-nanoparticle superlattices formed from anisotropic building blocks. Nat. Mater. 9, 913-917 (2010).

6. Senyuk, B. et al. Topological colloids. Nature 493, 200-205 (2013)

7. Poulin, P., Holger, S., Lubensky, T. C. \& Weitz, D. A. Novel colloidal interactions in anisotropic fluids. Science 275, 1770-1773 (1997)

8. Lapointe, C. P., Mason, T. G. \& Smalyukh, I. I. Shape-controlled colloidal interactions in nematic liquid crystals. Science 326, 1083-1086 (2009).

9. Ruhwandl, R. W. \& Terentjev, E. M. Long-range forces and aggregation of colloid particles in a nematic liquid crystal. Phys. Rev. E 55, 2958-2961 (1997).

10. Gu, Y. \& Abbott, N. L. Observation of Saturn-ring defects around solid microspheres in nematic liquid crystals. Phys. Rev. Lett. 85, 4719-4722 (2000).

11. Ramaswamy, S., Nityananda, R., Raghunathan, V. A. \& Prost, J. Power-law forces between particles in a nematic. Mol. Cryst. Liq. Cryst. Sci. Technol. Sect. A 288, 175-180 (1996).

12. Loudet, J. C. \& Poulin, P. Application of an electric field to colloidal particles suspended in a liquid-crystal solvent. Phys. Rev. Lett. 87, 165503 (2001).

13. Wood, T. A., Lintuvuori, J. S., Schofield, A. B., Marenduzzo, D. \& Poon, W. C. K. A self-quenched defect glass in a colloid-nematic liquid crystal composite. Science 334, 79-83 (2011).

14. Nelson, D. R. Toward a tetravalent chemistry of colloids. Nano. Lett. 2, 1125-1129 (2002).

15. Loudet, J. C., Barois, P. \& Poulin, P. Colloidal ordering from phase separation in a liquid- crystalline continuous phase. Nature 407, 611-613 (2000).

16. Ognysta, U. M. et al. Square colloidal lattices and pair interaction in a binary system of quadrupolar nematic colloids. Phys. Rev. E Stat. Nonlin. Soft Matter Phys. 83, 041709 (2011)

17. Liu, Q., Yuan, Y. \& Smalyukh, I. I. Electrically and optically tunable plasmonic guest-host liquid crystals with long-range ordered nanoparticles. Nano Lett. 14, 4071-4077 (2014)

18. Ramdane, O. O. et al. Memory-free conic anchoring of liquid crystals on a solid substrate. Phys. Rev. Lett. 84, 3871-3874 (2000).

19. Trivedi, R. P., Engström, D. \& Smalyukh, I. I. Optical manipulation of colloids and defect structures in anisotropic liquid crystal fluids. J. Optics 13, 044001 (2011).

20. Lee, T., Trivedi, R. P. \& Smalyukh, I. I. Multimodal nonlinear optical polarizing microscopy of long-range molecular order in liquid crystals. Opt. Lett. 35, 3447-3449 (2010).

21. Smalyukh, I. I., Kuzmin, A., Kachynski, A., Prasad, P. N. \& Lavrentovich, O. D. Optical trapping of colloidal particles and measurement of the defect line tension and colloidal forces in a thermotropic nematic liquid crystal. Appl. Phys. Lett. 86, 021913 (2005).

22. Stark, H. Physics of colloidal dispersions in nematic liquid crystals. Phys. Rep 351, 387-474 (2001).

23. Lubensky, T. C., Pettey, D., Currier, N. \& Stark, H. Topological defects and interactions in nematic emulsions. Phys. Rev. E 57, 610-625 (1998).

24. Chernyshuk, S. B. High-order elastic terms, boojums and general paradigm of the elastic interaction between colloidal particles in the nematic liquid crystals. Eur. Phys. J. E 37, 6 (2014).

25. Lev, B. I., Chernyshuk, S. B., Tomchuk, P. M. \& Yokoyama, H. Symmetry breaking and interaction of colloidal particles in nematic liquid crystals. Phys. Rev. E 65, 021709 (2002).

26. Tovkach, O. M., Chernyshuk, S. B. \& Lev, B. I. Theory of elastic interaction between arbitrary colloidal particles in confined nematic liquid crystals. Phys. Rev. E 86, 061703 (2012).

27. Muševič, I. Optical manipulation and self-assembly of nematic colloids: colloidal crystals and superstructures. Liq. Cryst. Today 9, 2-12 (2010).

28. Poulin, P. \& Weitz, D. A. Inverted and multiple nematic emulsions. Phys. Rev. E 57, 626-637 (1998)

29. Loudet, J. C., Hanusse, P. \& Poulin, P. Stokes drag on a sphere in a nematic liquid crystal. Science 306, 1525 (2004).

30. Stark, H. \& Ventzki, D. Stokes drag of spherical particles in a nematic environment at low Ericksen numbers. Phys. Rev. E 64, 031711 (2001).

31. Ruhwandl, R. W. \& Terentjev, E. M. Friction drag on a particle moving in a nematic liquid crystal. Phys. Rev. E 54, 5204-5210 (1996).

32. Ruhwandl, R. W. \& Terentjev, E. M. Monte Carlo simulation of topological defects in the nematic liquid crystal matrix around a spherical colloid particle. Phys. Rev. E 56, 5561-5565 (1997).

33. Chernyshuk, S. B., Tovkach, O. M. \& Lev, B. I. Elastic octopoles and colloidal structures in nematic liquid crystals. Phys. Rev. E 89, 032505 (2014).

34. Jackson, J. D. Classical Electrodynamics (John Wiley \& Sons, Inc., 1962).

35. Kaplan, I. G. Intermolecular Interactions: Physical Picture, Computational Methods and Model Potentials (John Wiley \& Sons, Ltd., 2006).

36. Ravnik, M., Alexander, G. P., Yeomans, J. M. \& Žumer, S. Three-dimensional colloidal crystals in liquid crystalline blue phases. Proc. Natl Acad. Sci. USA 108, 5188-5192 (2011).

37. Araki, T. \& Tanaka, H. Colloidal aggregation in a nematic liquid crystal: topological arrest of particles by a single-stroke disclination line. Phys. Rev. Lett. 97, 127801 (2006).

38. Giomi, L., Bowick, M. J., Ma, X. \& Majumdar, A. Molecular tilt on monolayerprotected nanoparticles. Europhys. Lett. 97, 36005 (2012).

39. Love, J. C., Estroff, L. A., Kriebel, J. K., Nuzzo, R. G. \& Whitesides, G. M. Self-assembled monolayers of thiolates on metals as a form of nanotechnology. Chem. Rev. 105, 1103-1169 (2005).

40. Liu, Q. et al. Plasmonic complex fluids of nematic-like and helicoidal selfassemblies of gold nanorods with negative order parameter. Phys. Rev. Lett. 109, 088301 (2012).

41. Lapointe, C. P., Hopkins, S., Mason, T. G. \& Smalyukh, I. I. Electrically-driven multi-axis rotational dynamics of colloidal platelets in nematic liquid crystals. Phys. Rev. Lett. 105, 178301 (2010).

42. Smalyukh, I. I., Butler, J., Shrout, J. D., Parsek, M. R. \& Wong, G. C. L. Elasticity-mediated nematic-like bacterial organization in model extracellular DNA matrix. Phys. Rev. E Stat. Nonlin. Soft Matter Phys. 78, 030701 (2008).

43. Senyuk, B. et al. Gourd-shaped colloidal particles in cholesteric liquid crystal. Soft Matter 10, 6014-6023 (2014).

44. Kim, J. W., Larsen, R. J. \& Weitz, D. A. Synthesis of nonspherical colloidal particles with anisotropic properties. J. Am. Chem. Soc. 128, 14374-14377 (2006).

45. Ma, F., Wang, S., Smith, L. \& Wu, N. Two-dimensional assembly of symmetric colloidal dimers under electric fields. Adv. Funct. Mater. 22, 4334-4343 (2012).

46. Wang, S., Ma, F., Zhao, H. \& Wu, N. Bulk synthesis of metal-organic hybrid dimers and their propulsion under electric fields. ACS Appl. Mater. Interfaces 6 , 4560-4569 (2014).

\section{Acknowledgements}

We thank T. Lubensky, S. Wang and N. Wu for discussions and acknowledge support of the National Science Foundation Grant DMR-1410735.

\section{Author contributions}

B.S. and O.P. performed the experimental work. B.S., O.M.T., S.B.C. and I.I.S. analysed the data. I.I.S. and B.S. wrote the manuscript with an input from all co-authors. I.I.S. conceived and directed the project.

\section{Additional information}

Supplementary Information accompanies this paper at http://www.nature.com/ naturecommunications 
Competing financial interests: The authors declare no competing financial interests.

Reprints and permission information is available online at http://npg.nature.com/ reprintsandpermissions/

How to cite this article: Senyuk, B. et al. Hexadecapolar colloids. Nat. Commun. 7:10659 doi: 10.1038/ncomms10659 (2016). (c) (i) This work is licensed under a Creative Commons Attribution 4.0 International License. The images or other third party material in this article are included in the article's Creative Commons license, unless indicated otherwise in the credit line; if the material is not included under the Creative Commons license, users will need to obtain permission from the license holder to reproduce the material. To view a copy of this license, visit http://creativecommons.org/licenses/by/4.0/ 